\title{
On critical exponents for the heat equation with a nonlinear boundary condition
}

by

\author{
Bei HU ${ }^{\dagger} *$ and Hong-Ming YIN $\dagger$ \\ $\dagger$ Department of Mathematics, University of Notre Dame, \\ Notre Dame, Indiana 46556.
}

ABSTRACT. - In this paper we consider the heat equation $u_{t}=\Delta u$ in a unbounded domain $\Omega \subset R^{N}$ with a Neumann boundary condition $u_{\nu}=u^{p}$, where $p>1$ and $\nu$ is the exterior unit normal on $\partial \Omega$. It is shown for various type of domains that there exists a critical number $p_{c}(\Omega) \geq 1$, such that all of positive solutions blow up in a finite time when $p \in\left(1, p_{c}\right]$ while there exist positive global solutions if $p>p_{c}$ and initial data are small.

\section{INTRODUCTION}

In this paper we consider the following problem:

$$
\begin{aligned}
\frac{\partial u}{\partial t} & =\Delta u \quad \text { for } x \in \Omega, t>0, \\
\frac{\partial u}{\partial \nu} & =u^{p} \quad \text { for } x \in \partial \Omega, t>0, \\
u(x, 0) & =u_{0}(x) \geq 0 \quad \text { for } x \in \Omega,
\end{aligned}
$$

* The work of this author is partially supported by National Science Foundation DMS 92 24935. 
where $\Omega$ is a (bounded or unbounded) connected domain in $R^{N}$ with boundary $\partial \Omega, \nu$ is the exterior normal vector on $\partial \Omega, p>1$ and $u_{0}(x) \geq 0$.

It is well known ( $c f$. [18]) that the solution of (1.1)-(1.3) will blow up in a finite time if initial datum is suitably large. An interesting question is what happens for small initial datum. Is there a Fujita type of critical exponent $p_{c}$ ? Namely, all positive solutions blow up in a finite time if the exponent $p \leq p_{c}$ while global solution exists if $p>p_{c}$ and initial value is small enough. As part of our motivation, we recall some interesting results on Fujita's critical exponents for equations with nonlinear reactions. Let $u(x, t)$ be a solution of the following problem:

$$
\begin{aligned}
& u_{t}=\Delta u+u^{p}, \quad \text { for } x \in R^{N}, t>0, \\
& u(x, 0)=u_{0}(x), \quad x \in R^{N} .
\end{aligned}
$$

Fujita [5] (see [19] for the case $p=p_{c}$ ) showed that $p_{c}=1+\frac{2}{N}$. When $R^{N}$ is replaced by $D_{k}=\left\{x=\left(x_{1}, \cdots, x_{N}\right) \in R^{N}: x_{1}>0, x_{2}>\right.$ $\left.0, \cdots, x_{k}>0\right\}, 1 \leq k \leq N$ with a supplement of zero Dirichlet boundary condition, then $p_{c}=1+\frac{2}{k+N}$ which depends on $k$ (see [13]). When $\Omega$ is a cone in $R^{N}, p_{c}=1+\frac{2}{2-\gamma}$, where $\gamma$ is the negative root of the equation $x(N-2+x)=\lambda_{1}$ while $\lambda_{1}$ is the first Dirichlet eigenvalue of LaplaceBeltrami operator on $\Omega \cap\{|x|=1\}$ (cf. [1]). In a recent paper [16], Ohta and Kaneko proved the following formula

$$
\frac{1}{p_{c}\left(D_{1} \times D_{2}\right)-1}=\frac{1}{p_{c}\left(D_{1}\right)-1}+\frac{1}{p_{c}\left(D_{2}\right)-1}
$$

for the product domain $\Omega=D_{1} \times D_{2}$. The reader can find an excellent survey [12] on this subject.

For the heat equation with a nonlinear boundary condition, there is a very nice survey [4] regarding the questions like blow up rate, blow up behavior, etc. Blow up rate, blow up set and asymptotic behavior has been studied in a number of papers. It has been shown under a very general condition that blow up can not occur at $t=\infty$ (see [14]). However, there has been not much progress for Fujita's type of critical exponent until very recently. When $\Omega$ is a bounded domain in $R^{N}$, it is known (cf. [3]) that all positive solutions blow up in a finite time (see also [10] for a short proof). Therefore, the critical number $p_{c}=\infty$ for a bounded domain. On the other hand, when $\Omega$ is a complement of a bounded domain, we will show (see Theorem 2.2 in Section 2) that the problem (1.1)-(1.3) always has global solutions if initial data are small when the dimension $N \geq 2$. It follows that the critical 
number $p_{c}=1$ when $N \geq 2$. These are two extreme cases. When a domain $\Omega$ has other general shape, the critical exponent depends on the domain. For the heat equation in $\Omega=R_{+}^{N}=\left\{x=\left(x_{1}, \cdots, x_{N}\right) \in R^{N}: x_{N}>0\right\}$ with $N \geq 2$, it is shown in [2] that $p_{c}=1+\frac{1}{N}$. The corresponding elliptic problem in the half space was studied in [9]. For the porous medium equation $u_{t}=\left(u^{m}\right)_{x x}$ in a half real line with boundary condition $u_{x}(0, t)=-u^{p}$, Galaktionov and Levine [6] proved that $p_{c}=m+1$, where $m \geq 1$.

In the present paper we study two types of domains. We show that if $\Omega$ is a convex cone type of domain in $R^{N}$ (see Definition (2.2)-(2.3) in Section 2) then $p_{c}(\Omega)=1+\frac{1}{N}$, in particular, $p_{c}\left(D_{k}\right)=1+\frac{1}{N}$, for all $1 \leq k \leq N-1$, which is interesting if one compares with the case of interior reaction. When $\Omega=R^{k} \times D$ where $D$ is a convex bounded domain in $R^{N-k}$ and $1 \leq k \leq N-1$, we prove that the critical exponent equals $1+\frac{2}{k}$.

To show the blow up of solutions, we first derive some properties of fundamental solution in convex domains and then prove the results by using integral inequalities. For global existence, we construct various auxiliary functions as supersolutions of (1.1)-(1.3). Those auxiliary functions are not trivial.

The paper is organized as follows. In Section 2, we state the main results. In Section 3, we state some propositions for the problem in a domain with uniformly Lipschitz continuous boundary. In Section 4, we show various blow up results. Global existence will be presented in Section 5 .

\section{THE MAIN RESULTS}

We begin with the case where $\Omega$ is a bounded smooth domain. It is known that all positive solutions blow up in a finite time if $1<p<\infty$. This result has been proved in [3]. We state this in the following theorem for completeness.

THEOREM 2.1. - The critical exponent $p_{c}(\Omega)=\infty$ if $\Omega$ is a bounded smooth domain; i.e., all positive solutions blow up in a finite time if $1<p<\infty$.

Next we consider the case when $\Omega$ is the complement of a bounded domain. As we mentioned in Introduction, for the connected complement of a bounded domain, the critical exponent is 1, i.e. there always exist positive solutions if initial values are small. Notice that when $N=1$, the complement of a bounded interval is two half spaces which is not 
connected. It is proved in [6] that for half-space, the critical exponent is 2. So we consider the case only for $N \geq 2$.

THEOREM 2.2. - Suppose that $\Omega$ is a connected complement of a bounded domain with smooth $\partial \Omega$. Then

$$
p_{c}(\Omega)=1
$$

i.e., if $p>1$, then there exist positive global solutions for all $0 \leq t<\infty$ provided that the initial data satisfy

$$
\begin{aligned}
& u_{0}(x) \leq \frac{\sigma}{|x|^{N-2}} \text { for } N \geq 3, \\
& u_{0}(x) \leq \sigma \exp \left(-|x|^{2}\right) \quad \text { for } N=2,
\end{aligned}
$$

where $\sigma$ is suitably small.

A bounded domain or the exterior of a bounded domain are two extreme cases. We next consider other intermediate domains.

Consider a convex cone type of domain, namely,

$$
\Omega=\left\{\left(x_{1}, x_{2}, \cdots, x_{N}\right) \mid x_{1}>G\left(x_{2}, \cdots, x_{N}\right)\right\},
$$

where we assume that $\Omega$ is convex and that there exists $M>0$ such that

$$
\begin{gathered}
G\left(x_{2}, \cdots, x_{N}\right) \geq 0 \\
\sum_{j=2}^{N}\left|\frac{\partial G}{\partial x_{j}}\right| \leq M \quad \text { for }\left(x_{2}, \cdots, x_{N}\right) \in R^{N-1} .
\end{gathered}
$$

THEOREM 2.3. - Let $\Omega$ be a convex domain satisfying the assumption (2.2)-(2.3). Then the critical exponent

$$
p_{c}(\Omega)=1+\frac{1}{N}
$$

in the following sense.

(i) If $1<p \leq p_{c}(\Omega)$, all positive solutions of the system (1.1)-(1.3) blow up in a finite time.

(ii) If $p>p_{c}(\Omega)$, then there exist solutions for all $0 \leq t<\infty$ provided that the initial data satisfy

$$
u_{0}(x) \leq \sigma \exp \left(-|x|^{2}\right) \quad \text { for } x \in \Omega
$$

where $\sigma$ is suitably small. 
Moreover, for any small $\varepsilon>0$, we can take the initial value small enough so that the global solutions decay to zero at the rate $t^{-\frac{N}{2}+\varepsilon}$.

With the appropriate change of coordinates, all convex cones

$$
\Omega=\{\lambda x ; \lambda>0, x \in D\}
$$

(where $D$ is an open convex set in $R^{N}$ such that $0 \notin D$ ) are included in Theorem 2.3. The domain $D_{k}$ discussed in Section 1 is a special case of the convex cone.

Next, we consider the case of a convex cylinder:

$$
\Omega=R^{k} \times D \quad(1 \leq k \leq N-1),
$$

where $D$ is a bounded, convex domain in $R^{N-k}$.

THEOREM 2.4. - The critical exponent

$$
p_{c}\left(R^{k} \times D\right)=1+\frac{2}{k},
$$

i.e.,

(i) If $1<p \leq p_{c}\left(R^{k} \times D\right)$, all positive solutions of the system (1.1)-(1.3) blow up in a finite time.

(ii) If $p>p_{c}\left(R^{k} \times D\right)$, then there exist solutions for all $0 \leq t<\infty$ provided that the initial data satisfy

$$
u_{0}(x) \leq \sigma \exp \left(-|x|^{2}\right) \text { for } x \in R^{k} \times D,
$$

where $\sigma$ is suitably small.

Moreover, the global solutions decay to zero at the rate $t^{-\frac{k}{2}}$ for small initial values.

\section{PRELIMINARIES}

In order to include those cones and $D_{k}$ (as mentioned in section 1) in our theorems, we need the existence and uniqueness for Lipschitz domains.

Definition. - We say that the domain $\Omega$ is uniformly Lipschitz, if the exterior and interior cone conditions are satisfied with a cone of fixed size for every $x \in \partial \Omega$.

Assume that $\Omega$ is a connected domain in $R^{N}$ with piecewise smooth and uniformly Lipschitz boundary $S=\partial \Omega$. We use the standard notation Vol. $13, n^{\circ} 6-1996$ 
$Q_{T}=\Omega \times(0, T)$. Since the boundary of the domain is not smooth, the classical theory of parabolic equations is not directly applicable. We shall state some propositions which will be used in the sequel. We shall first prove the comparison principle for Lipschitz domains.

Proposition 3.1. - Assume that $u_{1}(x, t), u_{2}(x, t)$ are two solutions for the system (1.1)-(1.3) such that

$$
\begin{aligned}
& u_{1}, u_{2} \in C^{2}\left(Q_{T}\right) \cap L^{2}\left(0, T ; H^{1}(\Omega \cap\{|x| \leq M\}) \text { for any } M>0,\right. \\
& u_{1}, u_{2} \in L^{\infty}\left(Q_{T}\right), \\
& \lim _{t \rightarrow 0}\left[u_{1}(x, t)-u_{2}(x, t)\right] \geq 0 \quad \text { for } x \in \Omega .
\end{aligned}
$$

Then

$$
u_{1}(x, t) \geq u_{2}(x, t) \text { for }(x, t) \in Q_{T} .
$$

Proof. - By regularity theory, the solutions $u_{1}$ and $u_{2}$ are smooth up to the smooth part of the boundary. The function $w(x, t)=u_{2}(x, t)-u_{1}(x, t)$ satisfies

$$
\begin{aligned}
& \frac{\partial w}{\partial t}=\Delta w \quad \text { for }(x, t) \in Q_{T}, \\
& \frac{\partial w}{\partial \nu}=u_{2}^{p}-u_{1}^{p} \quad \text { for }(x, t) \in S_{T}=\partial \Omega \times(0, T], \\
& w(x, 0)=0 \quad \text { for } x \in \Omega .
\end{aligned}
$$

Let $\zeta$ be a cut-off function such that

$$
0 \leq \zeta \leq 1, \quad \zeta(x)=0 \quad \text { for }|x|>M .
$$

Multiplying the equation by $\zeta^{2} w^{+}$and integrating over $\Omega$, we obtain

$$
\begin{aligned}
& \frac{\partial}{\partial t}\left(\int_{\Omega} \zeta^{2}\left(w^{+}\right)^{2} d x\right)+\int_{\Omega} \zeta^{2}\left|\nabla w^{+}\right|^{2} d x \\
& \quad \leq 4 \int_{\Omega}|\nabla \zeta|^{2}\left(w^{+}\right)^{2} d x+2 \int_{\partial \Omega} p u^{*} \zeta^{2}\left(w^{+}\right)^{2} d S_{x},
\end{aligned}
$$

where $u^{*}=\int_{0}^{1}\left[\theta u_{2}+(1-\theta) u_{1}\right]^{p-1} d \theta$.

Since $w(x, 0)=0$ and $u^{*}$ is bounded, the above inequality leads to

$$
\begin{aligned}
& \sup _{0 \leq \tau \leq t}\left[\int_{\Omega} \zeta^{2}(x, \tau)\left(w^{+}\right)^{2}(x, \tau) d x\right]+\int_{0}^{t} \int_{\Omega} \zeta^{2}\left|\nabla w^{+}\right|^{2} d x d t \\
& \quad \leq 4 \int_{0}^{t} \int_{\Omega}|\nabla \zeta|^{2}\left(w^{+}\right)^{2} d x d t+C \int_{0}^{t} \int_{\partial \Omega} \zeta^{2}\left(w^{+}\right)^{2} d S_{x} d t
\end{aligned}
$$


Since $\partial \Omega$ is uniformly Lipschitz, the Poincaré's inequality

$$
\int_{\partial \Omega} \varphi^{2} d S_{x} \leq \delta \int_{\Omega}|\nabla \varphi|^{2} d x+C(\delta) \int_{\Omega} \varphi^{2} d x \text { for all } \delta>0
$$

is valid for $\varphi \in H^{1}(\Omega)$ with the constant $C(\delta)$ depending only on $\Omega$ (see [15]). Letting $\varphi=\zeta \cdot w^{+}$in the above inequality and substituting into (3.1), we obtain

$$
\begin{aligned}
& \sup _{0 \leq \tau \leq t}\left[\int_{\Omega} \zeta^{2}(x, \tau)\left(w^{+}\right)^{2}(x, \tau) d x\right] \\
& \leq C \int_{0}^{t} \int_{\Omega}\left(\zeta^{2}+|\nabla \zeta|^{2}\right)\left(w^{+}\right)^{2} d x d t
\end{aligned}
$$

We now take smooth cut-off functions $\left\{\zeta_{\varepsilon}\right\}$ with compact support such that

$$
\begin{aligned}
& \zeta_{\varepsilon} \rightarrow \zeta_{0} \equiv \exp \left(-\sqrt{|x|^{2}+1}\right) \text { for } x \in \Omega, \\
& \nabla \zeta_{\varepsilon} \rightarrow \nabla \zeta_{0} \quad \text { for } x \in \Omega .
\end{aligned}
$$

Then $\left|\nabla \zeta_{0}\right| \leq \zeta_{0}$ and (3.2) implies that

$$
\begin{aligned}
& \sup _{0 \leq \tau \leq t}\left[\int_{\Omega} \zeta_{0}^{2}(x, \tau)\left(w^{+}\right)^{2}(x, \tau) d x\right] \\
& \leq C t \sup _{0 \leq \tau \leq t}\left[\int_{\Omega} \zeta_{0}^{2}(x, \tau)\left(w^{+}\right)^{2}(x, \tau) d x\right] .
\end{aligned}
$$

Thus $w^{+} \equiv 0$ if $t$ is small enough. The result now follows step-by-step to all $Q_{T} . \quad \square$ The comparison principle immediately implies the uniqueness of the system (1.1)-(1.3). In order to prove the local existence theorem for Lipschitz domains, we first establish some property of such domains.

Lemma 3.2. - Suppose that $\Omega$ is a uniformly Lipschitz domain in $R^{N}$. There exists a constant $c_{0}>0$, depending only $\partial \Omega$, such that for any $\varepsilon>0$, there exists a function $g_{\varepsilon}(x)$ with the following properties

$$
\begin{aligned}
& g_{\varepsilon} \in C^{\infty}\left(R^{N}\right) \\
& 0 \leq g_{\varepsilon}(x) \leq 1 \text { for } x \in \Omega \\
& 0 \leq g_{\varepsilon}(x) \leq \varepsilon \text { for } x \in \partial \Omega \\
& \frac{\partial g_{\varepsilon}}{\partial \nu} \geq c_{0} \text { for any } \nu \in N_{C}(x), x \in \partial \Omega \\
& \left|\Delta g_{\varepsilon}(x)\right| \leq C(\varepsilon) \text { for } x \in R^{N}
\end{aligned}
$$


where $N_{C}(x)$ is the normal cone, i.e.,

$$
N_{C}(x)=\left\{\nu ;|\nu|=1, \liminf _{y \rightarrow x, y \in \Omega} \frac{x-y}{|x-y|} \cdot \nu \geq 0\right\} .
$$

Proof. - We define

$$
g(x)=\left\{\begin{array}{ll}
\varepsilon & \text { for } x \in R^{N} \backslash \Omega, \operatorname{dist}(x, \partial \Omega) \geq \varepsilon \\
\operatorname{dist}(x, \partial \Omega) & \text { for } x \in R^{N} \backslash \Omega, \operatorname{dist}(x, \partial \Omega)<\varepsilon \\
-\operatorname{dist}(x, \partial \Omega) & \text { for } x \in \Omega, \operatorname{dist}(x, \partial \Omega)<\varepsilon \\
-\varepsilon & \text { for } x \in \Omega, \operatorname{dist}(x, \partial \Omega) \geq \varepsilon
\end{array} .\right.
$$

Let $\varphi_{\delta}(x)$ be the standard mollifier supported in $\{|x| \leq \delta\}$. If we take $\delta$ to be sufficiently small and $\delta<\varepsilon / 2$, then the function $g_{\varepsilon}(x)=\varepsilon+\varphi_{\delta} * g$ satisfies (3.3)-(3.7).

Proposition 3.3. - Assume that $u_{0}(x) \in C^{2}(\Omega) \cap L^{\infty}(\Omega) \cap H^{1}(\Omega \cap\{|x|<$ $M\}$ ) (for any $M>0$ ). Then there exists a small $T>0$ such that the problem (1.1)-(1.3) has a unique solution $u(x, t) \in C^{2,1}\left(Q_{T}\right) \bigcap L^{\infty}\left(Q_{T}\right)$ with $\nabla u$ being continuous up to the smooth part of the boundary. $u(x, t)$ satisfies the initial and boundary conditions (1.2)-(1.3) in the weak sense and pointwise except for those singular points on $S$.

Moreover, the solution can be extended in t direction as long as it remains finite in $L^{\infty}$ norm.

Proof. - When the boundary of $\Omega$ is smooth and $u_{0}$ is smooth and satisfying the compatibility condition, local existence of a classical solution in $C^{2+\alpha, 1+\frac{\alpha}{2}}\left(\bar{Q}_{T}\right) \cap L^{\infty}\left(Q_{T}\right)$ is elementary and can be established by, say, the contraction mapping principle (see [8]).

We now take $c_{0}$ as in Lemma 3.2, and let

$$
C_{1}=\frac{2}{c_{0}}\left(2+\left\|u_{0}\right\|_{L^{\infty}(\Omega)}\right)^{p}, \quad \varepsilon=\frac{1}{2 C_{1}} .
$$

Once $\varepsilon$ is fixed, we can take $g_{\varepsilon}$ as in Lemma 3.2 and let $C_{2}=$ $C_{1}\left\|\Delta g_{\varepsilon}\right\|_{L^{\infty}(\Omega)}$. Then the function

$$
\psi(x, t)=C_{1} g_{\varepsilon}(x)+C_{2} t+\left\|u_{0}\right\|_{L^{\infty}(\Omega)} \quad \text { for } 0<t<\frac{1}{C_{2}}
$$

is a local supersolution for (1.1)-(1.3). If we approximate the domain $\Omega$ by smooth $\Omega_{\lambda}$ such that $\Omega_{\lambda} \subset \Omega$, then for $\lambda$ sufficiently small,

$$
\begin{aligned}
& 0 \leq g_{\varepsilon}(x) \leq 1 \text { for } x \in \Omega_{\lambda}, \\
& 0 \leq g_{\varepsilon}(x) \leq 2 \varepsilon \text { for } x \in \partial \Omega_{\lambda}, \\
& \frac{\partial g_{\varepsilon}}{\partial \nu} \geq \frac{c_{0}}{2} \text { for } x \in \partial \Omega_{\lambda} .
\end{aligned}
$$


It follows that the function $\psi$ defined in (3.8) is a supersolution for the system (1.1)-(1.3) with $\Omega$ replaced by $\Omega_{\lambda}$, for all small $\lambda>0$ (remember that $\varepsilon$ is fixed). Therefore, for the approximation problem

$$
0 \leq u_{\lambda}(x, t) \leq \psi(x, t) \leq C_{1}+1+\left\|u_{0}\right\|_{L^{\infty}(\Omega)} \quad \text { for } x \in \Omega_{\lambda}, 0<t<\frac{1}{C_{2}}
$$

Using this uniform $L^{\infty}$-estimates, it is easy to show that

$$
\left\|u_{\lambda}\right\|_{L^{2}\left(0, T ; H^{1}(\Omega \cap\{|x| \leq M\})\right\}} \leq C(T, M) \text { for any } M>0
$$

where $C(T, M)$ is independent of $\lambda$ and $T=1 / C_{2}$. The compactness argument yields that the limit of a suitable subsequence of $\left\{u_{\lambda}\right\}$ exists and is a weak solution of the problem (1.1)-(1.3). The regularity theory of parabolic equations (cf. [11]) implies that $u(x, t)$ is of class $C^{2,1}\left(Q_{T}\right)$. Moreover, the solution $u(x, t)$ satisfies the initial and boundary conditions except at those points where the boundary of $\Omega$ is not differentiable ( $c f$. [11]). It is not hard to see that the solution can be extended in $t$ direction as long as it remains finite in $L^{\infty}$-norm.

\section{FINITE TIME BLOW UP}

To prove the theorems, we start with a general result which ensures the blow up of all solutions in a finite time if certain assumptions are satisfied.

Suppose that $\partial \Omega$ is piecewise smooth. Let $G_{N}(x, y, t, \tau)$ be the Green's function of the heat equation with homogeneous Neumann boundary condition. Assume that there exist constants $c_{0}>0, C_{1}>0,0 \leq \beta<1$ and nonnegative functions $\widetilde{G}(x, y, t-\tau), \varphi(x, t)$ with the following properties:

$$
\begin{aligned}
& G_{N}(x, y, t, \tau) \geq \widetilde{G}(x, y, t-\tau) \\
& \quad \text { for } 1<t<\infty, 0 \leq \tau<t, x, y \in \partial \Omega, \\
& c_{0} \leq \int_{\partial \Omega} \varphi(x, t) d S_{x} \leq C_{1} \quad \text { for } 1 \leq t<\infty \\
& \int_{\partial \Omega} t^{\beta} \widetilde{G}(x, y, t-\tau) \varphi(x, t) d S_{x} \geq c_{0} \varphi(y, \tau) \\
& \quad \text { for } y \in \partial \Omega, 2 \leq t<\infty, \frac{t}{2} \leq \tau \leq t .
\end{aligned}
$$


We further assume that there exist $c^{*}>0$ and $\eta>0$ such that

$$
\begin{aligned}
& \left\{\int_{\partial \Omega} \varphi(x, t)\left(\int_{\Omega} G_{N}(x, y, t, 0) u_{0}(y) d y\right)^{p} d S_{x}\right\}^{1 / p} \\
& \quad \geq c^{*} t^{-\eta} \text { for } t \gg 1
\end{aligned}
$$

THEOREM 4.1. - Under the assumptions (4.1)-(4.3), any positive solution of (1.1)-(1.3) with initial value satisfying (4.4) blows up in a finite time if

$$
p<1+\frac{1-\beta}{\eta} .
$$

In the case

$$
p=1+\frac{1-\beta}{\eta},
$$

the solution still blows up in a finite time if the initial datum satisfies (4.4) with the constant $c^{*}$ in (4.4) being large enough.

Proof. - In our proof, we shall use $\delta$ to denote various small positive generic constants depending only on $c_{0}, C_{1}$ and $p(\delta$ will be independent of $\left.c^{*}\right)$. We shall argue by contradiction. Assume that a positive solution of (1.1)-(1.3) exists for all $t>0$. Let $G_{N}$ be the Green's function for the heat equation with Neumann's boundary condition. Then

$$
\begin{aligned}
u(x, t)= & \int_{\Omega} G_{N}(x, y, t, 0) u_{0}(y) d y \\
& +\int_{0}^{t} \int_{\partial \Omega} G_{N}(x, y, t, \tau) u^{p}(y, \tau) d S_{y} d \tau \\
& \quad \text { for } x \in \bar{\Omega}, t>0
\end{aligned}
$$

We now take $T$ to be sufficiently large so that (4.4) is satisfied for $T / 2 \leq t \leq T(T \geq 2)$. We set

$$
\begin{gathered}
J_{0}(x, t)=\int_{\Omega} G_{N}(x, y, t, 0) u_{0}(y) d y \\
J_{1}(x, t)=\int_{T / 2}^{t} \int_{\partial \Omega} \widetilde{G}(x, y, t-\tau) u^{p}(y, \tau) d S_{y} d \tau .
\end{gathered}
$$


Then, by (4.1)

$$
u(x, t) \geq J_{0}(x, t)+J_{1}(x, t) .
$$

Clearly, from (4.4) for $t \in\left[\frac{T}{2}, T\right]$,

$$
\int_{\partial \Omega} \varphi(x, t) J_{0}^{p}(x, t) d S_{x} \geq \frac{c^{* p}}{T^{m p}} .
$$

Using (4.2), (4.3) and Jensen's inequality, we obtain,

$$
\begin{aligned}
& \int_{\partial \Omega} \varphi(x, t) J_{1}^{p}(x, t) d S_{x} \\
& \geq \delta\left\{\int_{T / 2}^{t} \int_{\partial \Omega} u^{p}(y, \tau) \int_{\partial \Omega} \tilde{G}(x, y, t-\tau) \varphi(x, t) d S_{x} d S_{y} d \tau\right\}^{p} \\
& \geq \delta\left\{\int_{T / 2}^{t} \frac{1}{t^{\beta}} \int_{\partial \Omega} u^{p}(y, \tau) \varphi(y, \tau) d S_{y} d \tau\right\}^{p} \\
& \geq \delta\left\{\int_{T / 2}^{t} \frac{1}{T^{\beta}} \int_{\partial \Omega} u^{p}(y, \tau) \varphi(y, \tau) d S_{y} d \tau\right\}^{p} \text { for } T / 2<t<T .
\end{aligned}
$$

Let

$$
A(t)=\left(\int_{\partial \Omega} u^{p}(x, t) \varphi(x, t) d S_{x}\right)^{1 / p} .
$$

Then for sufficiently large $T$,

$$
A^{p}(t) \geq \frac{c^{* p}}{T^{\eta p}}+\delta\left\{\int_{T / 2}^{t} \frac{A^{p}(\tau)}{T^{\beta}} d \tau\right\}^{p} \quad \text { for } T / 2<t \leq T,
$$

where we used the inequality

$$
(a+b)^{p} \geq a^{p}+b^{p} \quad \text { for } a \geq 0, b \geq 0, p \geq 1 .
$$

It follows that

$$
A(t) \geq \frac{1}{2}\left\{\frac{c^{*}}{T^{\eta}}+\delta \int_{T / 2}^{t} \frac{A^{p}(\tau)}{T^{\beta}} d \tau\right\} \text { for } T / 2<t \leq T,
$$

where we used the inequality

$$
a^{p}+b^{p} \geq \frac{(a+b)^{p}}{2^{p}} .
$$

Vol. $13, \mathrm{n}^{\circ}$ 6-1996. 
Let $g(t)$ denote the right-hand-side of the inequality (4.9), then

$$
g^{\prime}(t)=\frac{\delta}{2 T^{\beta}} A^{p}(t) \geq \frac{\delta}{2 T^{\beta}} g^{p}(t) \quad \text { for } T / 2<t \leq T .
$$

Integrating this inequality over $[T / 2, T]$, we obtain

$$
\frac{1}{p-1} g^{1-p}\left(\frac{T}{2}\right)>\frac{1}{p-1}\left[g^{1-p}\left(\frac{T}{2}\right)-g^{1-p}(T)\right] \geq \frac{\delta}{4} T^{1-\beta},
$$

i.e.,

$$
\frac{2^{(p-1)}}{(p-1) c^{*(p-1)}} T^{\eta(p-1)}>\frac{\delta}{4} T^{1-\beta} .
$$

This is a contradiction if $\eta(p-1)<1-\beta$ and $T$ is large enough.

It is clear from the above proof that the constant $\delta$ is independent of $c^{*}$ and $T$ (it depends only on $c_{0}, C_{1}$ and $p$ ). Therefore the proof is still valid for the case $\eta(p-1)=1-\beta$ if we take $c^{*}$ such that

$$
\frac{2^{(p-1)}}{(p-1) c^{*(p-1)}}=\frac{1}{2} \frac{\delta}{4}
$$

Any solution with initial datum satisfying (4.4) (with $c^{*}$ given above) still blows up in a finite time in this case.

Now we apply this theorem to show that for a large class of convex domains, the critical exponent is no less than $1+1 / N$.

LEMMA 4.2. - Let $\Omega$ be an unbounded convex domain with the property:

$$
\begin{aligned}
& \inf _{P \in \partial \Omega} \int_{\partial \Omega} \frac{1}{t^{(N-1) / 2}} \exp \left[-\left(\frac{p}{2}+1\right) \frac{|x-P|^{2}}{t}\right] d S_{x} \geq c \\
& \quad \text { for all } t>0 \text { and some } c>0 .
\end{aligned}
$$

If

$$
p \leq p_{c}(\Omega)=1+\frac{1}{N},
$$

then any positive solution of (1.1)-(1.3) blows up in a finite time.

Proof. - In the proof, we use $\delta$ for various generic positive constants, it may vary from one line to the other. Assume without loss of generality that $0 \in \partial \Omega$. We take

$$
\begin{aligned}
\widetilde{G}(x, y, t-\tau) & =\Gamma(x-y, t-\tau) \\
& \equiv\left(\frac{1}{4 \pi(t-\tau)}\right)^{\frac{N}{2}} \exp \left(-\frac{|x-y|^{2}}{4(t-\tau)}\right) . \\
\varphi(x, t) & =\frac{1}{t^{(N-1) / 2}} \exp \left(-\frac{|x|^{2}}{4 t}\right) .
\end{aligned}
$$


We shall next verify all assumptions of Theorem 4.1. The Green's function is constructed in the following way ( $c f$. [7]):

$$
G_{N}(x, y, t, \tau)=\Gamma(x-y, t-\tau)+h(x, y, t, \tau),
$$

where $h$ satisfies the backward heat equation with final datum

$$
\begin{aligned}
\left(\frac{\partial}{\partial \tau}+\Delta_{y}\right) h & =0 \quad \text { for } y \in \Omega, \tau<t \\
\frac{\partial h}{\partial \nu_{y}} & =-\frac{\partial \Gamma}{\partial \nu_{y}} \quad \text { for } y \in \partial \Omega, \tau<t, \\
h & =0 \quad \text { for } x \in \bar{\Omega}, t=\tau .
\end{aligned}
$$

Since we have a cylindrical domain and $\frac{\partial \Gamma}{\partial \nu_{y}}$ is a function of $x, y$ and $t-\tau$,

$$
G_{N}(x, y, t, \tau)=G_{N}(x, y, t-\tau, 0) \equiv G_{N}(x, y, t-\tau) .
$$

Since $\Omega$ is convex, $\frac{\partial \Gamma}{\partial \nu_{y}} \leq 0$ for $y \in \partial \Omega, x \in \bar{\Omega}$. It follows that $h \geq 0$ and

$$
G_{N}(x, y, t-\tau) \geq \Gamma(x-y, t-\tau) \quad \text { for } x, y \in \bar{\Omega}, \quad 0 \leq \tau<t \leq T .
$$

Thus (4.1) is valid, and

$$
\begin{aligned}
\int_{\partial \Omega} \Gamma(x-y, t) d S_{y} & =\frac{1}{(4 \pi)^{N / 2}} \frac{1}{\sqrt{t}} \int_{\partial \Omega} \exp \left(-\frac{|x-y|^{2}}{4 t}\right) \frac{d S_{y}}{t^{(N-1) / 2}} \\
& \equiv \frac{1}{(4 \pi)^{N / 2}} \frac{1}{\sqrt{t}} J(x, t) \quad \text { for } x \in \partial \Omega, 0<t<\infty
\end{aligned}
$$

where

$$
\begin{aligned}
& J(x, t)=\int_{\partial \Omega_{x, t}} \exp \left(-\frac{w^{2}}{4}\right) d S_{w} \\
& \left(\Omega_{x, t}=\{w ; \sqrt{t} w+x \in \Omega\}=\frac{\Omega-\{x\}}{\sqrt{t}}\right)
\end{aligned}
$$

is clearly bounded above and below (away from zero) uniformly in $x \in \partial \Omega$ and $t \geq 1$. In particular, (4.2) is satisfied (if we take $x=0$ ). To show (4.3) with $\beta=1 / 2$, we compute, for $t / 2<\tau<t$,

$$
\begin{aligned}
& \int_{\partial \Omega} \sqrt{t} \Gamma(x-y, t-\tau) \varphi(x, t) d S_{x} \\
& \quad \geq \int_{\partial \Omega} \sqrt{t-\tau} \Gamma(x-y, t-\tau) \varphi(x, t) d S_{x}
\end{aligned}
$$




$$
\begin{aligned}
= & \frac{1}{(4 \pi)^{N / 2}} \int_{\partial \Omega} \frac{1}{t^{(N-1) / 2}} \exp \left(-\frac{|x|^{2}}{4 t}\right) \\
& \times \exp \left(-\frac{|x-y|^{2}}{4(t-\tau)}\right) \frac{d S_{x}}{(t-\tau)^{(N-1) / 2}} \\
\geq & \frac{1}{(4 \pi)^{N / 2}}\left(\frac{1}{2}\right)^{(N-1) / 2} \varphi(y, \tau) \\
& \times \int_{\partial \Omega} \exp \left(\frac{|y|^{2}}{4 \tau}-\frac{|x|^{2}}{4 t}-\frac{|x-y|^{2}}{4(t-\tau)}\right) \frac{d S_{x}}{(t-\tau)^{(N-1) / 2}}
\end{aligned}
$$

Since

$$
\begin{aligned}
\frac{|y|^{2}}{4 \tau} & -\frac{|x|^{2}}{4 t}-\frac{|x-y|^{2}}{4(t-\tau)} \\
& \geq \frac{|y|^{2}}{4 \tau}-\frac{|x-y|^{2}+|y|^{2}+2|x-y||y|}{4 t}-\frac{|x-y|^{2}}{4(t-\tau)} \\
& =\frac{1}{4 t}\left(-2|x-y||y|+\frac{t-\tau}{\tau}|y|^{2}\right)-\frac{|x-y|^{2}}{4(t-\tau)}-\frac{|x-y|^{2}}{4 t} \\
& \geq-\frac{\tau|x-y|^{2}}{4 t(t-\tau)}-\frac{|x-y|^{2}}{4(t-\tau)}-\frac{|x-y|^{2}}{4 t} \\
& \geq-\frac{3|x-y|^{2}}{4(t-\tau)} \quad \text { for } 0<t / 2<\tau<t
\end{aligned}
$$

we derive from the assumption (4.11) that

$$
\begin{aligned}
& \int_{\partial \Omega} \exp \left(\frac{|y|^{2}}{4 \tau}-\frac{|x|^{2}}{4 t}-\frac{|x-y|^{2}}{4(t-\tau)}\right) \frac{d S_{x}}{(t-\tau)^{(N-1) / 2}} \geq \delta>0 \\
& \quad \text { for } 0<t / 2<\tau<t, \quad y \in \partial \Omega .
\end{aligned}
$$

Combining (4.18) and (4.19), we obtain (4.3).

Finally, by using $t=\varepsilon$ instead of $t=0$ if necessary, we may assume that $u_{0}(y)>0$ for all $y \in \bar{\Omega}$. Therefore

$$
\begin{aligned}
& \int_{\Omega} G_{N}(x, y, t, 0) u_{0}(y) d y \\
& \quad \geq \int_{\Omega} \Gamma(x-y, t) u_{0}(y) d y \\
& \quad \geq \int_{\Omega} \frac{1}{(4 \pi t)^{N / 2}} u_{0}(y) \exp \left(-\frac{|x|^{2}+|y|^{2}}{2 t}\right) d y \\
& \quad \geq \frac{1}{(4 \pi t)^{N / 2}} \exp \left(-\frac{|x|^{2}}{2 t}\right) \int_{\Omega} u_{0}(y) \exp \left(-|y|^{2}\right) d y
\end{aligned}
$$




$$
\begin{aligned}
& \geq \frac{c^{*}}{t^{N / 2}} \exp \left(-\frac{|x|^{2}}{2 t}\right) \\
& \quad \quad \text { for } 1<t<\infty, x \in \bar{\Omega}, \text { for some } c^{*}>0 .
\end{aligned}
$$

It follows that (4.4) is satisfied with $\eta=N / 2$. Thus by Theorem 4.1, all positive solutions blow up in a finite time if $p<1+1 / N$.

We now consider the critical case $p=1+1 / N$. Recall that $0 \in \partial \Omega$. From (4.20), we have

$$
u^{p}(x, t) \geq \frac{\delta^{p}}{t^{(N+1) / 2}} \exp \left(-\frac{p|x|^{2}}{2 t}\right) \quad \text { for } x \in \bar{\Omega}, t>1 .
$$

It follows from the representation (4.7) that

$$
\begin{aligned}
u(x, t) \geq & \delta \int_{1}^{t} \int_{\partial \Omega} \frac{1}{\tau^{(N+1) / 2}} \exp \left(-\frac{p|y|^{2}}{2 \tau}\right) \Gamma(x-y, t-\tau) d S_{y} d \tau \\
\geq & \delta \exp \left(-\frac{|x|^{2}}{t}\right) \int_{1}^{t / 2} \int_{\partial \Omega} \frac{1}{\tau^{(N+1) / 2}(t-\tau)^{N / 2}} \\
& \times \exp \left(\frac{|x|^{2}}{t}-\frac{p|y|^{2}}{2 \tau}-\frac{|x|^{2}+|y|^{2}}{2(t-\tau)}\right) d S_{y} d \tau \\
\geq & \frac{\delta}{t^{N / 2}} \exp \left(-\frac{|x|^{2}}{t}\right) \\
& \times \int_{1}^{t / 2} \int_{\partial \Omega} \frac{1}{\tau^{(N+1) / 2}} \exp \left(\frac{|x|^{2}}{t}-\frac{p|y|^{2}}{2 \tau}-\frac{|x|^{2}+|y|^{2}}{t}\right) d S_{y} d \tau \\
\geq & \frac{\delta}{t^{N / 2}} \exp \left(-\frac{|x|^{2}}{t}\right) \int_{1}^{t / 2} \frac{d \tau}{\tau} \\
\geq & \frac{\delta}{t^{N / 2}}\left(\log \frac{t}{2}\right) \exp \left(-\frac{|x|^{2}}{t}\right) \quad \text { for } x \in \bar{\Omega}, t>1 .
\end{aligned}
$$

Thus

$$
\begin{aligned}
& \int_{\Omega} G_{N}(x, y, t, 0) u\left(y, t_{0}\right) d y \\
& \quad \geq \int_{\Omega} \Gamma(x-y, t) u\left(y, t_{0}\right) d y \\
& \quad \geq \frac{\delta}{t_{0}^{N / 2}}\left(\log \frac{t_{0}}{2}\right) \int_{\Omega} \frac{1}{t^{N / 2}} \exp \left(-\frac{|x|^{2}+|y|^{2}}{2 t}\right) \exp \left(-\frac{|y|^{2}}{t_{0}}\right) d y \\
& \quad \geq \frac{1}{t^{N / 2}} \exp \left(-\frac{|x|^{2}}{2 t}\right) \frac{\delta}{t_{0}^{N / 2}}\left(\log \frac{t_{0}}{2}\right) \int_{\Omega} \exp \left(-\frac{|y|^{2}}{t_{0}}-\frac{|y|^{2}}{2 t}\right) d y \\
& \quad \geq \frac{1}{t^{N / 2}} \exp \left(-\frac{|x|^{2}}{2 t}\right) \delta\left(\log \frac{t_{0}}{2}\right) \quad \text { for } x \in \bar{\Omega}, 1<t_{0}<t<\infty,
\end{aligned}
$$

Vol. 13, n 6-1996. 
where the constant $\delta$ is independent of $t_{0}$. Therefore, if we use $t=t_{0}$ as the new initial value and take $t_{0}$ to be large enough, then the constant $c^{*}$ in (4.4) can be taken arbitrarily large. Therefore the solution blows up in a finite time in this case.

Remark 4.1. - Any domain satisfying (2.2) and (2.3) will satisfy (4.11).

Finally, we consider the case of a convex cylinder. Let $D$ be a bounded, convex domain in $R^{N-k}$, and let

$$
\Omega=R^{k} \times D \quad(1 \leq k \leq N-1) .
$$

LEMMA 4.3. - If

$$
p \leq p_{c}\left(R^{k} \times D\right)=1+\frac{2}{k}
$$

then any positive solution of (1.1)-(1.3) blows up in a finite time.

Proof. - We first derive some estimates for the Green's function $G_{N}(x, y, t-\tau)$. Clearly,

$$
\begin{aligned}
& \left(\frac{\partial}{\partial t}-\Delta_{y}\right) G_{N}(x, y, t)=0 \text { for } x \in R^{k} \times D, \quad t>1 \\
& \frac{\partial}{\partial \nu_{y}} G=0 \text { for } x \in R^{k} \times \partial D .
\end{aligned}
$$

Since $\Omega$ is convex,

(4.23) $G_{N}(x, y, t-\tau) \geq \Gamma(x-y, t-\tau)$ for $x, y \in \bar{\Omega}, 0<\tau<t<\infty$.

Especially,

$$
\begin{aligned}
& \left.G_{N}(x, y, t)\right|_{t=1} \\
& \quad \geq \exp \left(-\frac{|x-y|^{2}}{4}\right) \\
& \quad \geq \delta \exp \left(-\frac{\left|x^{\prime}-y^{\prime}\right|^{2}}{4}\right) \text { for } x, y \in R^{k} \times D \text { and some } \delta>0,
\end{aligned}
$$

where $x=\left(x_{1}, \cdots, x_{k}, x_{k+1}, \cdots, x_{N}\right)=\left(x^{\prime}, x_{k+1}, \cdots, x_{N}\right)$ and $y=$ $\left(y^{\prime}, y_{k+1}, \cdots, y_{N}\right)$. Therefore by comparison principle,

$$
G_{N}(x, y, t) \geq \frac{\delta}{t^{k / 2}} \exp \left(-\frac{\left|x^{\prime}-y^{\prime}\right|^{2}}{4 t}\right) \text { for } x, y \in R^{k} \times D, t>1 \text {. }
$$


So we take

$$
\begin{aligned}
\widetilde{G}(x, y, t) & =\left\{\begin{array}{l}
\frac{\delta}{t^{k / 2}} \exp \left(-\frac{\left|x^{\prime}-y^{\prime}\right|^{2}}{4 t}\right) \text { for } t>1 \\
\Gamma(x-y, t) \text { for } 0<t \leq 1
\end{array}\right. \\
\varphi(x, t) & =\frac{1}{t^{k / 2}} \exp \left(-\frac{\left|x^{\prime}\right|^{2}}{4 t}\right)
\end{aligned}
$$

It is clear that (4.2) and (4.3) are satisfied with $\beta=0$. Similar to the proof in Lemma 4.2, using (4.23) and (4.24), we derive (4.4) with $\eta=\frac{k}{2}$. Thus, by Theorem 4.1, any positive solution blows up in a finite time in the subcritical case $p<1+\frac{2}{k}$.

In the critical case $p=1+\frac{2}{k}$, we can use (4.24) to derive that

$$
u(x, t) \geq \frac{\delta}{t^{k / 2}}\left(\log \frac{t}{2}\right) \exp \left(-C \frac{\left|x^{\prime}\right|^{2}}{t}\right) \text { for } x \in R^{k} \times D
$$

This estimate implies that the constant $c^{*}$ in (4.4) can be chosen arbitrarily large in Theorem 4.1. The Lemma is proved.

\section{GLOBAL EXISTENCE}

A solution can be extended into a larger interval $\left[0, T^{*}\right]$ as long as we have an a priori estimate of $\|u\|_{L^{\infty}\left(Q_{T^{*}}\right)}$ (see Proposition 3.3). Therefore, the proof for global existence relies on deducing an a priori maximum norm of the solution.

We begin with the case where $\Omega$ is the connected complement of a bounded domain in $R^{N}$ with $\partial \Omega$ of class $C^{1}$. It should be pointed out that the associated elliptic problem admits a positive solution when $N \geq 3$. It is not hard to verify that when $N=2$ and $\Omega=R^{2} \backslash B_{1}(0)$, the associated elliptic problem (which reduces to an ODE) does not have any positive solutions.

Proof of Theorem 2.2. - We first consider the case where $N \geq 3$. It is not difficult to construct a supersolution in this case. Without loss of generality, we assume that $0 \notin \Omega$. Consider the following problem:

$$
\left\{\begin{array}{l}
\Delta v=0, \quad \text { for } x \in \Omega, \\
v(x)=1, \quad \text { for } x \in \partial \Omega \\
\lim _{|x| \rightarrow \infty} v(x)=0
\end{array}\right.
$$


To prove the existence of a positive solution for the above problem (5.1), we note that 0 is a subsolution while $\frac{C}{|x|^{N-2}}$ is a supersolution if $C$ is suitable large, where $|x|=\sqrt{x_{1}^{2}+\cdots+x_{N}^{2}}$. It follows from Theorem 8.2 of [17] (page 343) that the problem has a unique solution. As $v(x)$ takes positive maximum everywhere on $\partial \Omega$, the strong maximum principle implies $\partial v / \partial \nu>0$ for $x \in \partial \Omega$. Since $\partial \Omega$ is compact, $\partial v / \partial \nu \geq c_{0}>0$ on $\partial \Omega$, for some $c_{0}>0$, where $c_{0}$ depends on $\Omega$. If $\delta^{p-1} \leq c_{0}$, then the function $w(x)=\delta v(x)$ satisfies $w_{\nu}=\delta v_{\nu} \geq \delta c_{0} \geq w^{p}$ on $\partial \Omega$. Thus $w(x)$ is a supersolution of the problem (1.1)-(1.3) if $u_{0}(x) \leq w(x, 0)=\delta v(x)$. Therefore, $u(x, t)$ will be bounded by $\delta v(x)$ for $t>0$, if $u_{0}(x) \leq w(x, 0)$. This a priori estimate along with the local solvability ensures that the problem (1.1)-(1.3) has a solution in $\Omega \times[0, T]$ for any $T>0$.

For $N=2$, the system (5.1) does not have a solution. So we shall construct a supersolution in a different manner. For simplicity, we first consider the case that $\Omega^{c}$ is star shaped with respect to the origin 0 , i.e., $\nu \cdot x \leq-c_{0}<0$ on $\partial \Omega$. Let

$$
\begin{gathered}
\psi(x, t)=\frac{\varepsilon}{t^{1-\delta}} \exp \left(-\frac{|x|^{2}}{4 t}-\sum_{j=1}^{k} \frac{g_{j}(x)}{t^{\alpha_{j}}}\right), \\
\left(\alpha_{j}=2^{-j}, \quad 1 \leq j \leq k\right)
\end{gathered}
$$

where

$$
g_{1}(x)=\eta \sqrt{|x|}, \quad g_{2}(x)=\eta|x|^{1 / 4}, \cdots, g_{k}(x)=\eta|x|^{\alpha_{k}} .
$$

It is clear that

$$
x \cdot \nabla g_{j}(x)=\alpha_{j} g_{j}(x), \quad \Delta g_{j}(x)=\frac{\eta \alpha_{j}^{2}}{|x|^{2-\alpha_{j}}} \geq \frac{4 \eta \alpha_{j+1}^{2}}{|x|^{2-\alpha_{j}}}=\frac{4}{\eta}\left|\nabla g_{j+1}\right|^{2} .
$$

It follows that

$$
\begin{aligned}
\psi_{t}-\Delta \psi & \\
=\psi \cdot & {\left[-\frac{1-\delta}{t}+\frac{|x|^{2}}{4 t^{2}}+\sum_{j=1}^{k} \frac{\alpha_{j} g_{j}}{t^{\alpha_{j}+1}}\right.} \\
& \left.+\frac{1}{t}+\sum_{j=1}^{k} \frac{\Delta g_{j}}{t^{\alpha_{j}}}-\sum_{i=1}^{2}\left(\frac{x_{i}}{2 t}+\sum_{j=1}^{k} \frac{1}{t^{\alpha_{j}}} \frac{\partial g_{j}}{\partial x_{i}}\right)^{2}\right] \\
\geq \psi \cdot & {\left[\frac{\delta}{t}+\sum_{j=1}^{k}\left(\frac{\alpha_{j} g_{j}}{t^{\alpha_{j}+1}}-\frac{1}{t^{\alpha_{j}+1}} \sum_{i=1}^{2} x_{i} \frac{\partial g_{j}}{\partial x_{i}}\right)\right.} \\
& \left.+\sum_{j=1}^{k} \frac{\Delta g_{j}}{t^{\alpha_{j}}}-\sum_{i=1}^{2}\left(\sum_{j=1}^{k} \frac{1}{t^{\alpha_{j}}} \frac{\partial g_{j}}{\partial x_{i}}\right)^{2}\right]
\end{aligned}
$$




$$
\begin{aligned}
& \geq \psi \cdot\left[\frac{\delta}{t}+\frac{4}{\eta} \sum_{j=1}^{k-1} \frac{\left|\nabla g_{j+1}\right|^{2}}{t^{\alpha_{j}}}+\frac{\Delta g_{k}}{t^{\alpha_{k}}}-k \sum_{j=1}^{k} \frac{1}{t^{2 \alpha_{j}}}\left|\nabla g_{j}\right|^{2}\right] \\
& =\psi \cdot\left[\frac{1}{t}\left(\delta-k\left|\nabla g_{1}\right|^{2}\right)+\sum_{j=1}^{k-1}\left(\frac{4}{\eta}-k\right) \frac{\left|\nabla g_{j+1}\right|^{2}}{t^{\alpha_{j}}}+\frac{\Delta g_{k}}{t^{\alpha_{k}}}\right] \\
& >0,
\end{aligned}
$$

provided we take $\eta$ such that

$$
\eta \leq \min \left[2 \sqrt{\frac{\delta}{k} \min _{x \in \bar{\Omega}}|x|}, \frac{4}{k}\right]
$$

We take $k$ such that $p \geq 1+\alpha_{k} /(1-\delta)$ (this is possible since $p>1$ ). Then

$$
\frac{\partial \psi}{\partial \nu} \geq \frac{\varepsilon \psi}{t^{\alpha_{k}}} \alpha_{k}|x|^{\alpha_{k}-1}(-\nu \cdot x)>\psi^{p} \quad \text { for } x \in \partial \Omega, t>1
$$

provided $\varepsilon^{p-1}<c_{0} \alpha_{k} \min _{x \in \partial \Omega}|x|^{\alpha_{k}-1}$. It follows that if initial value satisfies

$$
u_{0}(x) \leq \psi\left(x, t_{0}\right) \text { for } x \in \Omega \text { for some } t_{0} \geq 1
$$

then the solution exists globally in time, with $u(x, t) \leq \psi\left(x, t+t_{0}\right)$ for all $0<t<\infty$.

We next consider a general exterior domain without the star shape assumption. Assume that $0 \notin \Omega$. We shall still define $\psi$ as in (5.2) with

$$
g_{1}(x)=\eta \sqrt{|x|}, \quad \cdots, g_{k-1}(x)=\eta|x|^{\alpha_{k-1}},
$$

and $g_{k}(x)$ to be determined. As before, we can choose $\eta$ so that $\delta / 2>k\left|\nabla g_{1}(x)\right|^{2}$. Then the previous calculation shows that

$$
\psi_{t}-\Delta \psi \geq \psi \cdot\left[\frac{\delta}{2 t}-\frac{1}{t^{1+\alpha_{k}}} x \cdot \nabla g_{k}+\frac{\Delta g_{k-1}}{t^{\alpha_{k-1}}}+\frac{\Delta g_{k}}{t^{\alpha_{k}}}-\frac{k}{t^{2 \alpha_{k}}}\left|\nabla g_{k}\right|^{2}\right]
$$

Therefore, if $g_{k}$ satisfies

$$
\left\{\begin{array}{l}
g_{k} \geq 0, \quad \Delta g_{k} \geq 0 \quad \text { for } x \in \Omega \\
\left|\nabla g_{k}\right| \leq \frac{\widetilde{\varepsilon}}{|x|} \quad \text { for } x \in \Omega \\
\frac{\partial g_{k}}{\partial \nu} \leq-\widetilde{\varepsilon}<0 \quad \text { for } x \in \partial \Omega, \quad \text { for some } \eta>0
\end{array}\right.
$$


then $\psi_{t}-\Delta \psi>0$ for $t>1$ provided $\widetilde{\varepsilon}$ is small enough. We shall prove later that such $g_{k}(x)$ exists. As before, take $k$ such that $p \geq 1+\alpha_{k} /(1-\delta)$. Then

$$
\frac{\partial \psi}{\partial \nu} \geq \varepsilon \psi \cdot\left(-\frac{1}{t^{\alpha_{k}}} \frac{\partial g_{k}}{\partial \nu}-C \sum_{j=1}^{k-1} \frac{1}{t^{\alpha_{j}}}\right)>\psi^{p} \quad \text { for } x \in \partial \Omega, t>t^{*}
$$

provided $\varepsilon$ is sufficiently small and $t^{*}$ is sufficiently large. Therefore if initial datum satisfies

$$
u_{0}(x) \leq \psi\left(x, t_{0}\right) \quad \text { for } x \in \Omega \quad \text { for some } t_{0} \geq t^{*},
$$

then the solution of (1.1)-(1.3) exists globally in time.

To complete the proof, we now show the existence of the function in (5.6). Take $R>0$ to be large enough such that $\Omega^{c} \subset \subset B_{R}(0)$. Let $h(x)$ be a solution of the following problem

$$
\begin{aligned}
& \Delta h=0 \text { for } x \in \Omega \cap B_{R}(0), \\
& h=0 \text { for } x \in \partial \Omega, \\
& h=1 \text { for } x \in \partial B_{R}(0) .
\end{aligned}
$$

It is clear that

$$
0 \leq h(x) \leq 1 \quad \text { for } x \in \Omega \cap B_{R}(0), \quad \frac{\partial h}{\partial \nu} \leq-c<0 \quad \text { for } x \in \partial \Omega .
$$

We now let

$$
g_{k}(x)=\left\{\begin{array}{ll}
\tilde{\delta} h(x) & \text { for } x \in \Omega \cap \overline{B_{R}(0)} \\
\widetilde{\delta}\left[\bar{C} \log \left(\frac{|x|}{R}\right)+1\right] & \text { for }|x| \geq R
\end{array} \quad(\delta>0) .\right.
$$

If we choose $\bar{C} \geq R \max _{|x|=R}|\nabla h(x)|$, then

$$
\Delta g_{k} \geq 0 \text { for } x \in \Omega \text { in the distribution sense. }
$$

We next take $\widetilde{\delta}$ to be small enough, then all requirements in (5.6) are satisfied. The Theorem is proved.

We next consider the domain with the following property:

There exist $P \in R^{N} \backslash \Omega, g(x)$ and $\eta>0,0 \leq \theta \leq 1$ such that

$$
\begin{gathered}
\frac{\partial}{\partial \nu}\left(|x-P|^{2}\right) \leq 0 \quad \text { for } x \in \partial \Omega, \\
g(x) \geq 0, \quad \Delta g \geq 0, \quad|\nabla g(x)| \leq 1+|x-P|^{\theta} \quad \text { for } x \in \Omega, \\
\frac{\partial g(x)}{\partial \nu} \leq-\eta<0 \text { for } x \in \partial \Omega .
\end{gathered}
$$


Lemma 5.1. - Let the assumption (5.8) be in force. Suppose that

$$
p>p_{c}(\Omega)=1+\frac{1+\theta}{N}
$$

Then there exist solutions of (1.1)-(1.3) for all $t \in[0, \infty)$, if the initial value $u_{0}(x)$ satisfies

$$
u_{0}(x) \leq \sigma \exp \left(-|x|^{2}\right) \quad \text { for } x \in \Omega,
$$

for a suitably small $\sigma>0$.

Proof. - We shall construct a supersolution of the form

$$
\psi(x, t)=\frac{\varepsilon}{t^{-\delta+N / 2}} \exp \left[-\left(1-\frac{\delta}{N}\right) \frac{|x-P|^{2}}{4 t}-\frac{g_{1}(x)}{t^{(1+\theta) / 2}}\right],
$$

where $g_{1}(x)=\widetilde{\varepsilon} g(x), \widetilde{\varepsilon}$ is small and $g(x)$ is given in (5.8). It is clear that

$$
\begin{aligned}
& \mid\left(1-\frac{\delta}{N}\right) \frac{(x-P) \cdot \nabla g_{1}}{t^{(3+\theta) / 2} \mid \leq} \frac{\delta}{2 N}\left(1-\frac{\delta}{N}\right) \frac{|x-P|^{2}}{4 t^{2}} \\
&+2\left(\frac{N}{\delta}-1\right) \frac{\left|\nabla g_{1}\right|^{2}}{t^{1+\theta}} \\
& \frac{1+|x-P|^{2 \theta}}{t^{\theta}} \leq 2+\frac{|x-P|^{2}}{t} \text { for } t \geq 1 .
\end{aligned}
$$

It follows that

$$
\begin{aligned}
& \psi_{t}-\Delta \psi \\
& =\psi \cdot\left\{\frac{\delta}{2 t}+\frac{\delta}{N}\left(1-\frac{\delta}{N}\right) \frac{|x-P|^{2}}{4 t^{2}}+\frac{(1+\theta) g_{1}(x)}{2 t^{(3+\theta) / 2}}+\frac{\Delta g_{1}}{t^{(1+\theta) / 2}}\right. \\
& \left.-\frac{\left|\nabla g_{1}\right|^{2}}{t^{1+\theta}}-\left(1-\frac{\delta}{N}\right) \frac{(x-P) \cdot \nabla g_{1}}{t^{(3+\theta) / 2}}\right\} \\
& \geq \psi \cdot\left\{\frac{\delta}{2 t}+\frac{\delta}{2 N}\left(1-\frac{\delta}{N}\right) \frac{|x-P|^{2}}{4 t^{2}}-\frac{2 N}{\delta} \frac{\left|\nabla g_{1}\right|^{2}}{t^{1+\theta}}\right\} \\
& \geq \frac{\psi}{t}\left\{\frac{\delta}{2}+\frac{\delta}{2 N}\left(1-\frac{\delta}{N}\right) \frac{|x-P|^{2}}{4 t}-\frac{4 N(\widetilde{\varepsilon})^{2}}{\delta}\left(\frac{1+|x-P|^{2 \theta}}{t^{\theta}}\right)\right\} \\
& \geq 0 \text { for } x \in \Omega, t>1 \text {, }
\end{aligned}
$$

provided $\widetilde{\varepsilon}$ is small enough. We fix such an $\widetilde{\varepsilon}$. Since $p>1+(1+\theta) / N$, we can choose $\delta$ so that

$$
\left(\frac{N}{2}-\delta\right)+\frac{1+\theta}{2} \leq p\left(\frac{N}{2}-\delta\right)
$$

Vol. $13, \mathrm{n}^{\circ} 6-1996$ 
Thus, by (5.8),

$$
\frac{\partial \psi}{\partial \nu} \geq \psi^{p} \quad \text { for } x \in \partial \Omega, t>1
$$

provided

$$
\varepsilon^{p-1} \leq \widetilde{\varepsilon \eta}
$$

It follows that if initial datum satisfies

$$
u_{0}(x) \leq \psi\left(x, t_{0}\right) \quad \text { for } x \in \Omega \text { for some } t_{0}>1,
$$

then the solution exists globally in time.

It turns out that there is a large class of domains which satisfy the assumption (5.8). Let

$$
\Omega=\left\{\left(x_{1}, x_{2}, \cdots, x_{N}\right) \mid x_{1}>G\left(x_{2}, \cdots, x_{N}\right)\right\},
$$

where we assume that there exists $M>0$ such that

$$
\begin{gathered}
G\left(x_{2}, \cdots, x_{N}\right) \geq 0 \\
\sum_{j=2}^{N}\left|\frac{\partial G}{\partial x_{j}}\right| \leq M \quad \text { for }\left(x_{2}, \cdots, x_{N}\right) \in R^{N-1},
\end{gathered}
$$

$$
x_{2} G_{x_{2}}+\cdots+x_{N} G_{x_{N}} \leq G+M \quad \text { for }\left(x_{2}, \cdots, x_{N}\right) \in R^{N-1} .
$$

LEMMA 5.2. - If $\Omega$ is given by (5.13) with $G$ satisfying (5.14)-(5.15), then the assumption (5.8) is satisfied with $\theta=0$. Therefore, there exist solutions to (1.1)-(1.3) for all $t \in[0, \infty)$, if

$$
p>1+1 / N
$$

and the initial value $u_{0}(x)$ satisfies

$$
u_{0}(x) \leq \sigma \exp \left(-|x|^{2}\right) \text { for } x \in \Omega,
$$

for a suitably small $\sigma>0$.

Proof. - The exterior normal direction on $\partial \Omega$ is given by

$$
\nu=\frac{\left(-1, \nabla^{\prime} G\right)}{\sqrt{1+\left|\nabla^{\prime} G\right|^{2}}}
$$

where $\nabla^{\prime}=\left(\frac{\partial}{\partial x_{2}}, \cdots, \frac{\partial}{\partial x_{N}}\right)$. 
If we take $g(x)=x_{1}$, then

$$
\frac{\partial g(x)}{\partial \nu}=-\frac{1}{\sqrt{1+\left|\nabla^{\prime} G\right|^{2}}} \leq \frac{-1}{\sqrt{1+M^{2}}} .
$$

It is also clear that all remaining conditions on $g(x)$ in (5.8) are satisfied with $\theta=0$.

We now take $P=(-M, 0,0, \cdots, 0)$, then

$$
\frac{\partial}{\partial \nu}|x-P|^{2}=2 \frac{-(G+M)+x_{2} G_{x_{2}}+\cdots+x_{N} G_{x_{N}}}{\sqrt{1+\mid \nabla^{\prime} G^{2}}} \leq 0 .
$$

Thus the lemma follows from Lemma 5.1.

Remark 5.1. - If $\Omega$ is convex, then (5.14) is satisfied with $M=0$.

Remark 5.2. - Any convex cone will satisfy (5.14)-(5.15). Because of the constant $M,(5.14)-(5.15)$ are the assumptions for the domain at $x=\infty$. Roughly speaking, (5.14) requires the growth of $G$ at infinity no more than linear growth and (5.15) requires some kind of convexity of the domain near infinity.

We next replace (5.14) with

$$
\begin{gathered}
G\left(x^{\prime}\right) \geq 0 \\
\sum_{j=2}^{N}\left|\frac{\partial G}{\partial x_{j}}\right| \leq M\left(1+G^{\theta}\right) \quad \text { for } x^{\prime}=\left(x_{2}, \cdots, x_{N}\right) \in R^{N-1} .
\end{gathered}
$$

where $0 \leq \theta \leq 1$.

LEMMA 5.3. - If $\Omega$ is given by (5.13) with $G$ satisfying (5.14a) and (5.15), then the assumption (5.8) is satisfied. Therefore, there exist solutions to (1.1)-(1.3) for all $t \in[0, \infty)$, if

$$
p>1+(1+\theta) / N
$$

and the initial value $u_{0}(x)$ satisfies

$$
u_{0}(x) \leq \sigma \exp \left(-|x|^{2}\right) \quad \text { for } x \in \Omega,
$$

for a suitably small $\sigma>0$.

Proof. - If we take $g(x)=x_{1}+x_{1}^{1+\theta}$, then

$$
\frac{\partial g(x)}{\partial \nu}=-\frac{1+(1+\theta) G^{\theta}}{\sqrt{1+\left|\nabla^{\prime} G\right|^{2}}} \leq-\eta \quad \text { for } x \in \partial \Omega
$$


for some $\eta>0$. It is also clear that all remaining conditions on $g(x)$ in (5.8) are satisfied. Thus the lemma follows from Lemma 5.1.

Remark 5.3. - Lemma 5.3 includes all domains of the type $\left\{\left(x_{1}, \cdots, x_{N}\right) ; x_{1}>G\left(x_{2}, \cdots, x_{N}\right)\right\}$ where $G$ is given by

$$
\begin{gathered}
G\left(x^{\prime}\right)=\left[\sqrt{b_{0}+b_{2} x_{2}^{2}+b_{3} x_{3}^{2}+\cdots+b_{N} x_{N}^{2}}\right]^{1 /(1-\theta)}, \\
b_{0} \geq 0, b_{2} \geq 0, \cdots, b_{N} \geq 0,
\end{gathered}
$$

for $0 \leq \theta<1$, or

$$
\begin{gathered}
G\left(x^{\prime}\right)=\exp \left[\sqrt{b_{0}+b_{2} x_{2}^{2}+b_{3} x_{3}^{2}+\cdots+b_{N} x_{N}^{2}}\right], \\
b_{0} \geq 0, b_{2} \geq 0, \cdots, b_{N} \geq 0,
\end{gathered}
$$

for $\theta=1$.

Finally, we consider a cylinder $\Omega=R^{k} \times D$, where $D$ is a bounded domain in $R^{N-k}$ with uniformly Lipschitz boundary $\partial D$.

Lemma 5.4. - For $\Omega=R^{k} \times D$, there exist solutions of (1.1)-(1.3) for all $t \in[0, \infty)$, if

$$
p>p_{c}(\Omega)=1+\frac{2}{k},
$$

and the initial value $u_{0}(x)$ satisfies

$$
u_{0}(x) \leq \sigma \exp \left(-|x|^{2}\right) \quad \text { for } x \in \Omega,
$$

for a suitably small $\sigma>0$.

Proof. - We shall construct a supersolution of the form

$$
=\frac{\varepsilon}{t^{k / 2}}\left(M+\frac{g\left(x_{k+1}, \cdots, x_{N}\right)}{t^{1+\delta}}\right) \exp \left(-\frac{1}{t^{\delta}}\right) \exp \left(-\frac{\left|x^{\prime}\right|^{2}}{4 t}\right),
$$

where $x^{\prime}=\left(x_{1}, \cdots, x_{k}\right)$. We take $g$ to be a smooth function on $\bar{D}$ such that,

$$
\begin{aligned}
& g\left(x_{k+1}, \cdots, x_{N}\right) \geq 0 \text { in } D, \\
& \frac{\partial g}{\partial \nu}\left(x_{k+1}, \cdots, x_{N}\right) \geq 1 \text { on } \partial D \text {, }
\end{aligned}
$$


where $\nu$ is the exterior normal on $\partial D$. A direct calculation shows that

$$
\begin{aligned}
& \left(\frac{\partial}{\partial t}-\Delta\right) \psi \\
& =\frac{\varepsilon}{t^{k / 2}} \exp \left(-\frac{1}{t^{\delta}}\right) \exp \left(-\frac{\left|x^{\prime}\right|^{2}}{4 t}\right) \\
& \quad \times\left\{\frac{\delta}{t^{1+\delta}}\left(M+\frac{g\left(x_{k+1}, \cdots, x_{N}\right)}{t^{1+\delta}}\right)-\frac{(1+\delta) g}{t^{2+\delta}}-\frac{\Delta g}{t^{1+\delta}}\right\} \\
& >0 \quad \text { for } x \in R^{k} \times D, t>1,
\end{aligned}
$$

if we take

$$
M=\frac{1}{\delta}\left(\|\Delta g\|_{L^{\infty}(D)}+(1+\delta)\|g\|_{L^{\infty}(D)}\right) .
$$

On the boundary,

$$
\begin{aligned}
\frac{\partial \psi}{\partial \nu} & =\frac{\varepsilon}{t^{k / 2}}\left(\frac{1}{t^{1+\delta}}\right) \exp \left(-\frac{1}{t^{\delta}}\right) \exp \left(-\frac{\left|x^{\prime}\right|^{2}}{4 t}\right) \\
& \geq \frac{\varepsilon}{t^{1+\delta+k / 2}} \exp \left(-\frac{1}{t^{\delta}}\right) \exp \left(-\frac{\left|x^{\prime}\right|^{2}}{4 t}\right) \\
& \geq \psi^{p} \quad \text { for } x \in R^{k} \times \partial D, t>1,
\end{aligned}
$$

provided we take

$$
\delta=\frac{k}{2}\left(p-1-\frac{2}{k}\right), \quad \varepsilon^{p-1} \leq \frac{1}{\left[M+\|g\|_{L^{\infty}(D)}\right]^{p}}
$$

It follows that if initial datum satisfies

$$
u_{0}(x) \leq \psi\left(x, t_{0}\right) \text { for } x \in R^{k} \times D \text { for some } t_{0}>1
$$

then the solution exists globally in time.

Finally, Theorem 2.3 is a combination of Lemma 4.2 and Lemma 5.2 while Theorem 2.4 is a combination of Lemma 4.3 and Lemma 5.4.

Remark 5.4. - No convexity assumption is needed for the domain $D$ for the proof of global existence.

\section{ACKNOWLEDGEMENT}

The authors are very grateful to the referee for the helpful suggestions. 


\section{REFERENCES}

[1] C. BANDLE and H. A. LEvine, On the existence and nonexistence of global solutions of reaction-diffusion equations in sectorial domains, Trans. Amer. Math. Soc., Vol. 316, 1989, pp. 595-624.

[2] K. Deng, M. FIl.a and H. A. Levine, On critical exponents for a system of heat equations coupled in the boundary conditions, Acta Math. Univ. Comenianae, Vol. 63, 1994, pp. 169-192.

[3] M. FILA, Boundedness of global solutions for the heat equation with nonlinear boundary conditions, Comment. Math. Univ. Carolinate, Vol. 30, 1989, pp. 479-484.

[4] M. FILA and J. FILo, Blow-up on the boundary: a survey, Proceedings of the Banach Center, to appear.

[5] H. Fuנrta, On the blowing up of solutions of the Cauchy problem for $u_{t}=\Delta u+u^{1+\alpha}$, J. Fac, Sci. Univ. Tokyo Sect. A. Math., Vol. 16, 1966, pp. 105-113.

[6] V. A. Galaktionov and H. A. Levine, On critical Fujita exponents for heat equations with a nonlinear flux boundary condition on the boundary, Israel J. Math., to appear.

[7] M. G. Garroni and J. L. Menaldi, Green functions for second order parabolic integrodifferential problems, Longman Scientific and Technical, New York, 1992.

[8] J. L. Gomez, V. Marquez and N. Wolanski, Blow up and localization of blow up points for the heat equation with a nonlinear boundary condition, J. Diff. Eq., Vol. 2, 1992, pp. 384-401.

[9] B. Hu, Nonexistence of a positive solution of the Laplace equation with a nonlinear boundary condition, Differential and Integral Equations, Vol. 7, 1994, pp. 301-313.

[10] B. Hu and H.-M. YIN, The profile near blow up time for solutions of the heat equation with a nonlinear boundary condition, Trans. Amer. Math. Soc., Vol. 346, 1994, pp. I17-135.

[11] O. A. Ladyzenskaja, V. A. Solonnikov and N. N. Ural'ceva, Linear and Quasilinear Equations of Parabolic type, AMS Monograph translation, Vol. 23, Providence, RI, 1968.

[12] H. A. LEviNE, The role of critical exponents in blow up theorems, SIAM Review, Vol. 32 , 1990 , pp. 262-288.

[13] H. A. Levine and P. MEIER, The value of the critical exponent for reaction-diffusion equations in cones, Arch. Rational Mech. Anal., Vol. 109, 1990, pp. 73-80.

[14] G. Lieberman, Study of global solutions of parabolic equations via a priori estimates, Part I: Equations with principal elliptic part equal to the Laplacian, Math. Meth. in the Appl. Sci., Vol. 16, 1993, pp. 457-474.

[15] V. G. MaZ'ja, Sobolev Spaces, Springer-Verlag, New York, 1985.

[16] S. OHTA and A. KanEKo, Critical exponent of blow up for semilinear heat equation on a product domain, J. Fac. Sci. Univ. Tokyo, Sect. IA, Math., Vol. 40, 1993, pp. 635-650.

[17] C. V. PAo, Nonlinear Parabolic Equation and Elliptic Equations, Plenum Press, New York, 1992.

[18] W. WALTER, On existence and nonexistence in the large of solutions of parabolic differential equations with a nonlinear boundary condition, SIAM J. Math. Anal., Vol. 6, 1975 , pp. 85-90.

[19] F. B. WEISSLER, Existence and nonexistence of global solutions for a semilinear heat equation, Israel J. Math., Vol. 38, 1981, pp. 29-40.

(Manuscript received February 6, 1995.) 